\title{
Vertical silicon nanowire-based racetrack resonator optical sensor
}

\author{
S. M. Sherif ${ }^{1}$ (D) M. Y. Elsayed ${ }^{2} \cdot$ L. A. Shahada ${ }^{1} \cdot$ M. A. Swillam $^{2}$
}

Received: 31 October 2018 / Accepted: 15 August 2019 / Published online: 21 October 2019

(c) The Author(s) 2019

\begin{abstract}
We propose a highly sensitive optical sensor which is built from silicon nanowires. The silicon nanowires are arranged to form a ring resonator. The silicon nanowires cladding and voids are filled with the analyte. The sensor has a small footprint of $16 \mu \mathrm{m} \times 16.5 \mu \mathrm{m}$. The insertion loss of the sensor is only $0.4 \mathrm{~dB}$, while it is characterized by its high sensitivity of $430 \mathrm{~nm} /$ RIU. As a biosensor, our device showed a $100 \mathrm{~nm} /$ RIU sensitivity when a thin biolayer of $10 \mathrm{~nm}$ thickness is attached to the silicon nanowire structures.
\end{abstract}

\section{Introduction}

Silicon on insulator "SOI" waveguides are the most commonly used type of waveguides in photonic components and devices because of their low losses and complementary metal oxide semiconductor "CMOS" fabrication compatibility $[1,2]$. In SOI waveguides, the majority of the photonic mode is confined in the silicon core, while an exponentially decaying evanescent tail extends through the cladding, quantified by the confinement factor [3]. Thus, the effective refractive index of the guided mode is defined by the silicon core index with minimum contribution from the surrounding medium. However, in applications such as optical modulators and sensors, it is usually needed to increase the overlap between the photonic mode and the surrounding medium "cladding". In this context, it is reasonable to discuss the plasmonic $[4,5]$ and silicon nanowires "SiNWs" platforms $[6,7]$.

The plasmonic platform enables the confinement and enhancement of fields at metal-dielectric interfaces, and in slot waveguides [8]. Thus, plasmonic sensors benefit from the strong interaction between the effective mode and the

S. M. Sherif

sherifms@qu.edu.qa

M. A. Swillam

m.swillam@aucegypt.edu

1 Department of Chemistry and Earth Sciences, College of Arts and Sciences, Qatar University, P.O. Box 2713, Doha, Qatar

2 Department of Physics, School of Sciences and Engineering, The American University in Cairo, New Cairo 11835, Egypt analyte in contact with the metal surface [9]. The SiNWs platform, which is built of arrays of SiNWs on an insulator substrate, is characterized by having voids between the SiNWs [10]. So, SiNWs sensors benefit from the diffusion of the analyte through these voids, as well as the cladding.

However, the SiNWs platform has two main advantages when compared with the plasmonic platform. First, plasmonics are usually associated with high losses due to the large free electron density in metals and doped semiconductors [11], such high losses are not observed in SiNWs. Second, in contrast to plasmonic materials, SiNWs are CMOS compatible. This enables the integration of the photonic and electronic components on the same chip, since they are both fabricated by the CMOS fabrication technology [12].

Moreover, various applications have been developed using the SiNWs platform such as routers [13], and sensors [14]. The propagation loss of SiNWs waveguides is negligible when compared with the bend loss. Since at sharp bends, the electromagnetic energy may escape through the poorly guiding voids, different parameters of SiNWs should be carefully studied such as the nanowire diameter, pitch, height, and bend radius [15].

Hereby, we demonstrate a fully compatible CMOS optical sensor. The sensor is based on the ring resonator mechanism. The sensor is built of SiNW arrays on $\mathrm{SiO}_{2}$ substrate, the SiNWs are arranged to form a ring structure. A commercialgrade simulator eigenmode solver and propagator [16] was used to analyze the modes and effective index. A 3D simulator based on the finite difference time domain method [17] was used to study the sensor performance in terms of its spectrum, extinction ratio, insertion loss, resonator Q-factor, and sensitivity. 

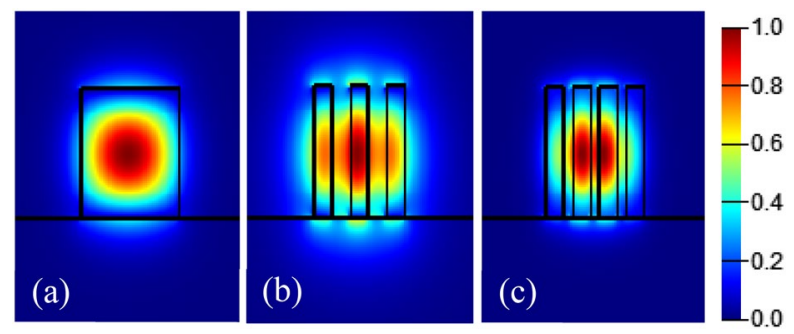

Fig. 1 Electric field distribution $(\operatorname{Re}\{E z\})$ for a conventional SOI, $\mathbf{b}$ design $\mathrm{A}$, and $\mathbf{c}$ design $\mathrm{B}$

\section{SiNWs and SOI waveguides}

In our previous work on SiNWs waveguides, we have developed a directional coupler-based optical router [13]. The insertion loss of that device was limited to $1.3 \mathrm{~dB}$. The cross-section of the router was designed using 3 SiNWs, with equal diameter $100 \mathrm{~nm}$, pitch $200 \mathrm{~nm}$, and height of $600 \mathrm{~nm}$. For convention, we will call this platform "design A" while our newly proposed platform in this paper is "design B", as shown in Fig. 1. In the directional coupler, there were no worries about the bend loss, since the directional coupler had no bends. However, in this present work, we are building a SiNWs sensor which utilizes the ring resonator mechanism. Thus, bend loss should be taken into account.

In general, we may need to increase the Si existence and reduce free space (voids), to reduce the radiation of optical power at the bends, i.e. decreases the bend loss. In particular, we may need to increase the number, diameter of the SiNWs, and/or decrease the pitch. So, we need a compromise between the bend loss, and the sensitivity of the sensor. On one hand, increasing the voids and reducing $\mathrm{Si}$ results in higher interaction of the photonic mode with the analyte which leads to higher sensitivity, but higher bend loss. On the other hand, decreasing the voids and increasing the Si results in lower bend loss, but it decreases the sensitivity of the sensor.

The height of $600 \mathrm{~nm}$ remained constant between design A and design B. Shorter nanowires are more difficult to fabricate using the suggested fabrication methods (see Sect. 3.2). Longer nanowires would require the use of a taller input waveguide, which would likely support more than one mode, which is undesirable. We start the optimization of the sensor by considering design A, however, the large voids of $100 \mathrm{~nm}$ results in high radiation at the bends, with minimal optical power detected at the output such that the insertion loss reaches $5 \mathrm{~dB}$, such design is inefficient for its huge power loss Thus, it is obvious that we need to increase the Si content on the expense of the voids between the SiNWs. Therefore we decreased the

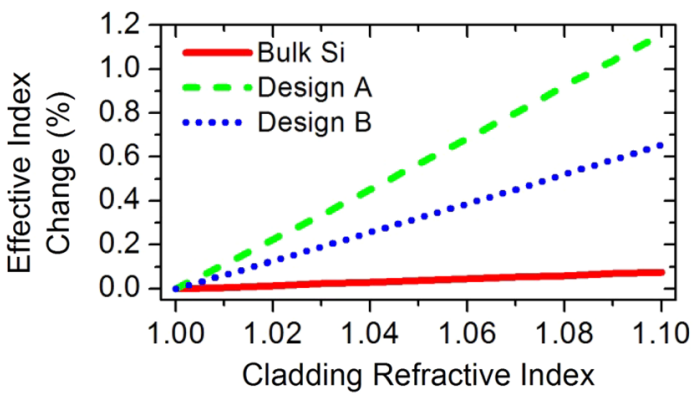

Fig. 2 Effective index percentage change with the change in the cladding (analyte) index at $1.55 \mu \mathrm{m}$ wavelength

pitch from 200 to $150 \mathrm{~nm}$, such that the distance between two adjacent nanowires is $50 \mathrm{~nm}$, and we increased the number of SiNWs from 3 to 4 as shown in Fig. 1c.

We further investigate one more design (design C) of four SiNWs and $25 \mathrm{~nm}$ spacing between two adjacent nanowires, this design results in a small insertion loss of $0.4 \mathrm{~dB}$. However, design $\mathrm{C}$ is challenging in terms of fabrication feasibility because of its small spacing of $25 \mathrm{~nm}$, and also its measured sensitivity is limited to $400 \mathrm{~nm} / \mathrm{RIU}$ which is less than that of design B $(430 \mathrm{~nm} / \mathrm{RIU})$ as will be discussed later.

The sensitivity of the sensor is strongly dependent on the interaction of the effective mode with the analyte. This can be quantified by measuring the effective index change in response to changes in the analyte index. The electric field distribution within the SOI, design A, design B waveguides are shown in Fig. 1. Figure 2 shows that the effective index response to the analyte index change is minimal for the SOI as expected, while it is higher for design A than design B.

\section{SiNW ring resonator}

\subsection{Device structure}

Figure $3 \mathrm{a}$ shows the $3 \mathrm{D}$ design of the SiNW ring resonator, while Fig. $3 \mathrm{~b}$ shows the cross-section of the resonator. The refractive indices used are 1.45 for $\mathrm{SiO}_{2}$, and 3.45 for $\mathrm{Si}$, while the voids and cladding index is initially set to 1 at $\lambda=1.55 \mu \mathrm{m}$. The bus waveguide width and height are $100 \mathrm{~nm}$, and $600 \mathrm{~nm}$, respectively. The thickness of the $\mathrm{SiO}_{2}$ is $3 \mu \mathrm{m}$.

Figure 4 shows the electric field confinement in the resonators built from both designs. Design A needed a racetrack resonator structure of $6 \mu \mathrm{m}$ coupling length to achieve critical coupling to the resonator. It can be observed that design A suffers from power leakage at the bends, while design B strongly guide the optical power through the bends. Quantitatively, at $1.55 \mu \mathrm{m}$ wavelength, the insertion loss of Design 


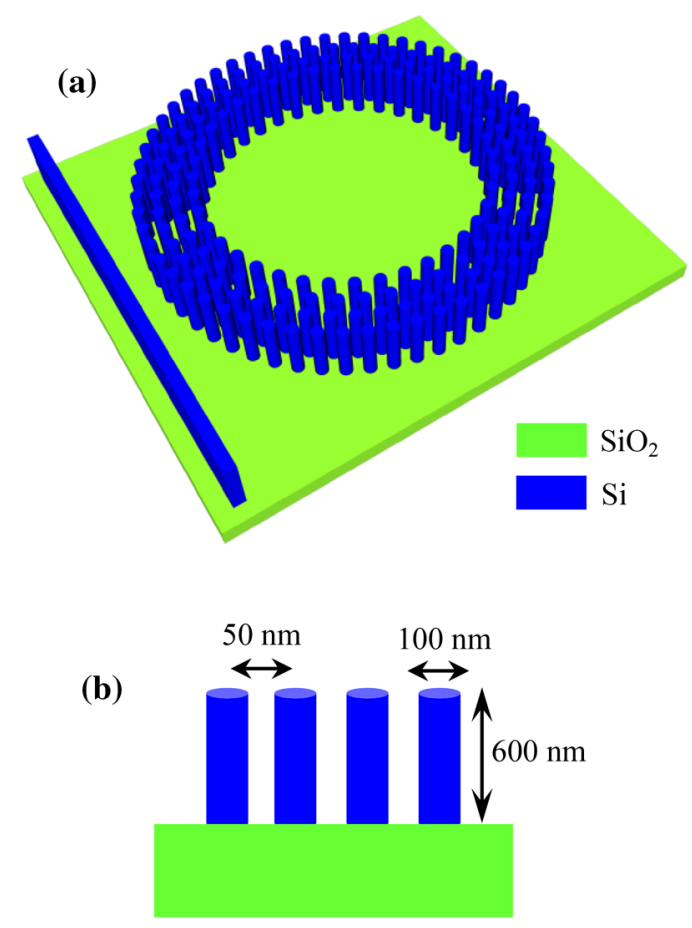

Fig. 3 Schematic layout of the SiNWs ring resonator sensor: a perspective view and $\mathbf{b}$ cross-section view
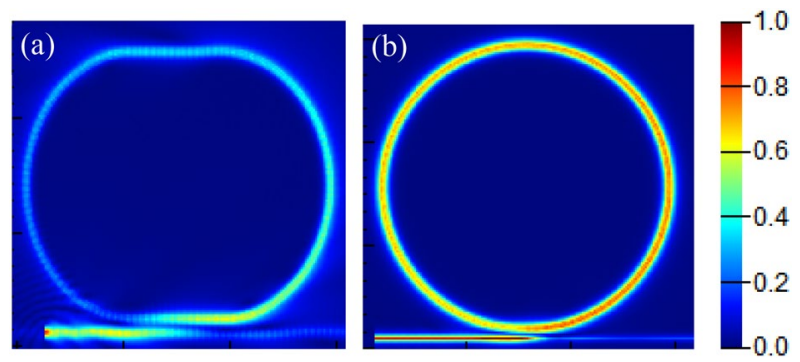

Fig. 4 Electric field distribution $\left|E_{\mathrm{z}}\right|$ in the ring resonators: a design A: 3 nanowires of $100 \mathrm{~nm}$ diameter each, and $100 \mathrm{~nm}$ spacing, and b design B: 4 nanowires of $100 \mathrm{~nm}$ diameter each and $50 \mathrm{~nm}$ spacing

A is $3 \mathrm{~dB}$, while that of Design B is only $0.4 \mathrm{~dB}$. Thus, design $\mathrm{B}$ was favored over design $\mathrm{A}$ because of the high bend loss and insertion loss of design $\mathrm{A}$.

\subsection{Fabrication procedure}

This section suggests different fabrication methods. Silicon nanowires are patterned on a SOI wafer with device layer of $600 \mathrm{~nm}$ height using E-beam lithography or nanosphere lithography, followed by deep reactive ion etching $[18,19]$ as shown in Fig. 5.

Figure 5a shows the typical E-beam lithography sequence and reactive ion etching. Figure $5 \mathrm{~b}$ shows lithography steps that are achievable using deep UV lithography without resorting to complicated double patterning techniques, arriving at a ring that is based on regular solid SOI waveguide. Figure $5 \mathrm{c}$ shows how to convert the waveguide to be based on vertical nanowires instead of the solid waveguide. Alternatively, it is possible to convert the ring that is based on regular solid waveguide to the vertical nanowire platform by replacing the nanosphere lithography step in Fig. 5b with metal-assisted chemical etching (MACE) or laser excimer method. Figure 6 shows exemplary SEM images of such nanowires from our preliminary fabrication trials using the fabrication protocols detailed at [20-22] and [23] for MACE and laser excimer, respectively. In Fig. 6a, the oxide layer is clearly visible.

In Fig. 6b, a regular n-type silicon substrate was used.

\section{Results and discussion}

\subsection{Device optimization}

The bus waveguide is excited with a TM wave, the effective index measured is 1.66. The optical power couples from the bus waveguide to the ring resonator. Figure 7 a shows the extinction ratio as a function of the gap distance between the bus waveguide and the ring resonator, at a ring radius of $5 \mu \mathrm{m}\left(\mathrm{ER}=10 \log \left(1 / P_{\text {out }}\right)\right)$, where $P_{\text {out }}$ is the output power.

Also, the $Q$-factor of the resonator was calculated for different gaps, $Q=\lambda / \Delta \lambda$, where $\Delta \lambda$ is the full width at half maximum of the $1.55 \mu \mathrm{m}$ resonance. For gaps larger than $200 \mathrm{~nm}$, the $Q$-factor increases as shown in Fig. 7b, but their corresponding ER increases. In other words, as the gap increases, the resonances become sharper, while less power is coupled to the resonator. Thus, the gap was set to $200 \mathrm{~nm}$ to achieve critical coupling, resulting in a $Q$-factor of 310 .

The insertion loss of the sensor, shown in Fig. 8, is the sum of the bend loss and the propagation loss. The propagation loss can be neglected when compared with the bend loss because the structure does not include lossy materials. Small bend radii result in smaller footprint of the device, while it results in higher bend loss. Larger bend radii significantly reduce the bend loss, but result in larger device footprint. Thus, we choose a bend radius of $8 \mu \mathrm{m}$, which has an insertion loss of $0.4 \mathrm{~dB}$, and footprint of $16 \mu \mathrm{m} \times 16.5 \mu \mathrm{m}$.

\subsection{Sensitivity measurements}

The ring resonator has its resonances at distinct wavelength positions given by:

$\lambda_{m}=\frac{2 \pi \cdot r \cdot n_{e f f}}{m}$ 

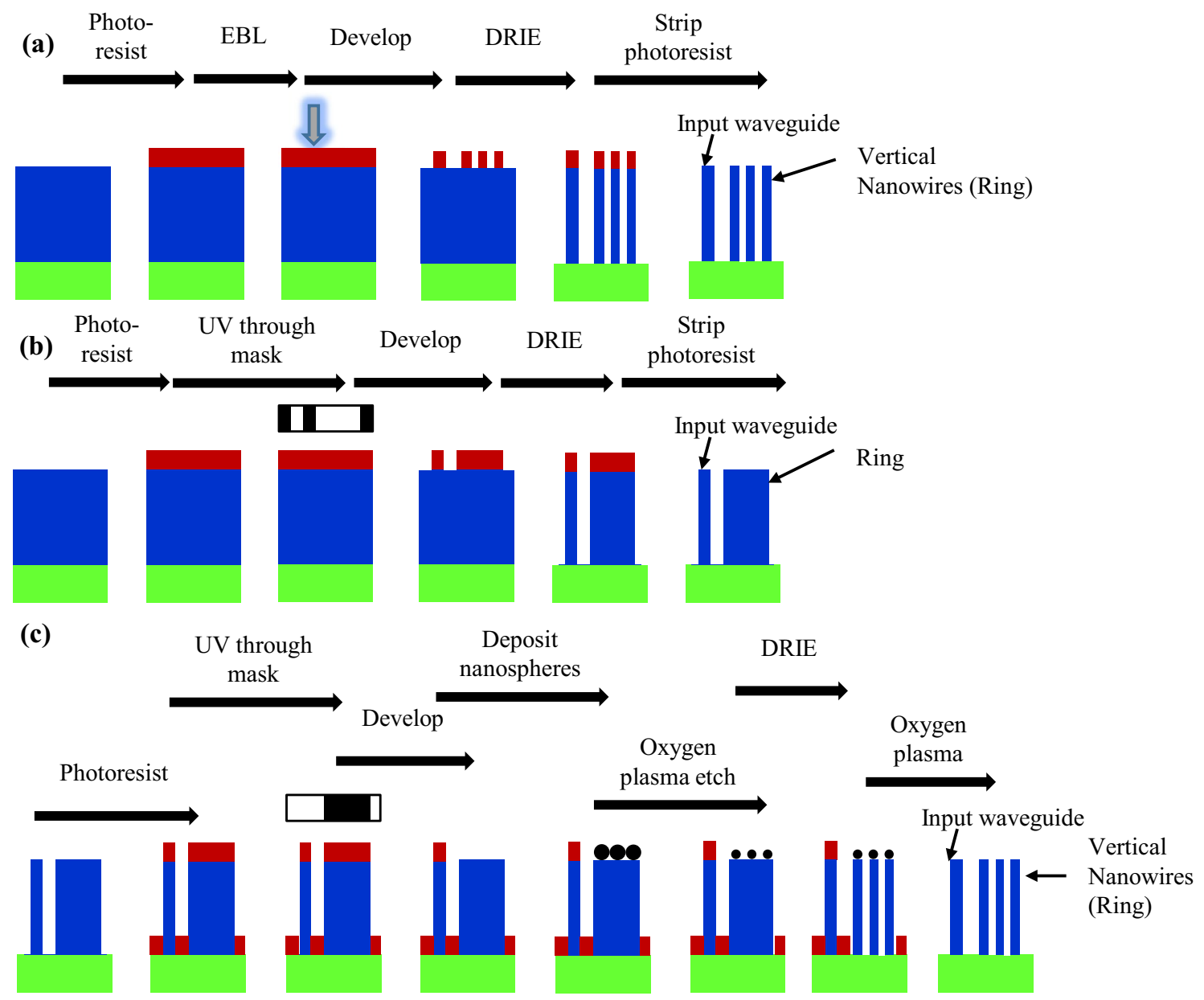

Fig. 5 Suggested fabrication methods. a Electron-beam lithography, b UV lithography to define the input waveguide and ring resonator, $\mathbf{c}$ nanosphere lithography to convert the ring resonator waveguide platform to the vertical nanowire platform

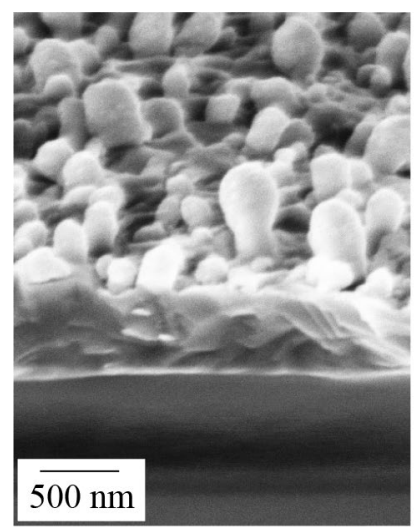

(a)

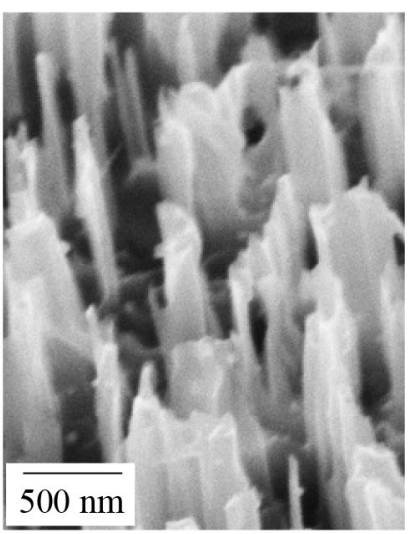

(b)
Fig. 6 Scanning electron microscope (SEM) images of nanowires fabricated by a the laser excimer method on a SOI wafer, $\mathbf{b}$ metalassisted chemical etching
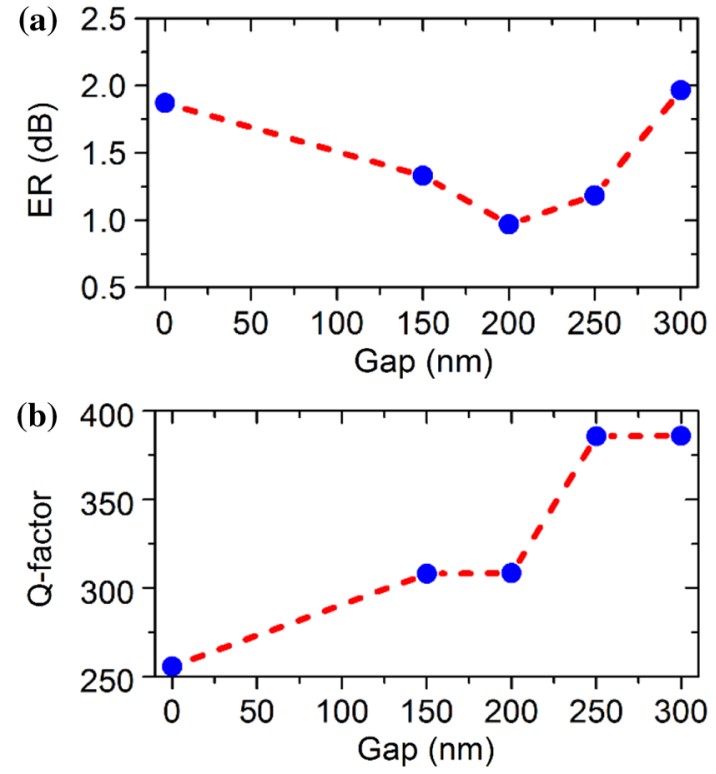

Fig. 7 Gap distance effect on the a extinction ratio and $\mathbf{b} Q$-factor at $\lambda=1.55 \mu \mathrm{m}$ 


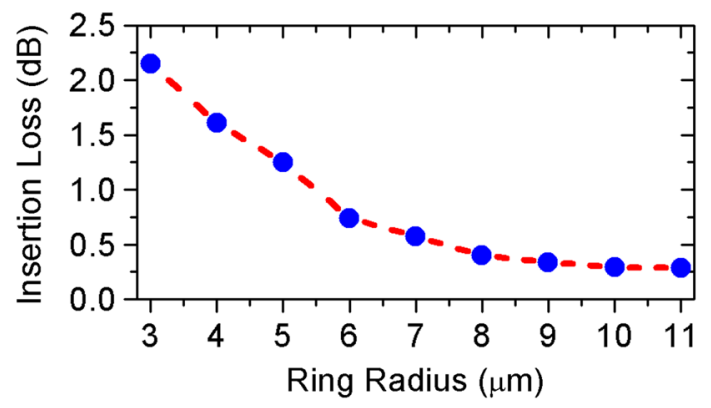

Fig. 8 Evolution of the insertion loss of the sensor with the ring radius at $\lambda=1.55 \mu \mathrm{m}$

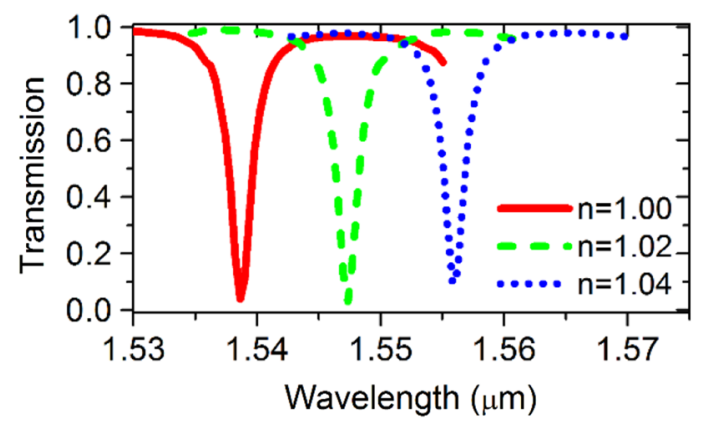

Fig. 9 Spectrum red-shift due to increasing the surrounding index

where $m$ is the resonance order, $\lambda$ is the wavelength position, $r$ is the ring radius, $n_{\text {eff }}$ is the effective index. When the surrounding index changes which could be due to the diffusion of gases or smokes, the effective index changes leading to a shift in the resonance wavelength as given by (1), and shown in the spectrum of Fig. 9.

For gas sensing applications, the index of the voids and cladding was gradually increased from 1.00 to 1.05 while recording the wavelength position. This simulates the diffusion of mixtures of gases with different indices through the voids and cladding. Sensor sensitivity of $430 \mathrm{~nm} / \mathrm{RIU}$ is calculated from the slope of the curve of Fig. 10a. Moreover, the sensor sensitivity was studied in the analyte index range of 1.33-1.37 to account for water and biological materials sensing, the slope of Fig. 10b results in a sensitivity of $420 \mathrm{~nm} /$ RIU.

Previously reported SiNWs sensors [14] used a ring resonator design that resulted on $243 \mathrm{~nm} / \mathrm{RIU}$ sensitivity, where the number, diameter, pitch, height of the SiNWs were 9, $50 \mathrm{~nm}, 75 \mathrm{~nm}$, and $700 \mathrm{~nm}$, respectively. However, our sensor achieved a higher sensitivity of $430 \mathrm{~nm} / \mathrm{RIU}$.

\subsection{Biosensing applications}

Biosensing applications are vital in the photonics research community, where a vast number of biosensors were developed using different platforms such as the silicon nanowire
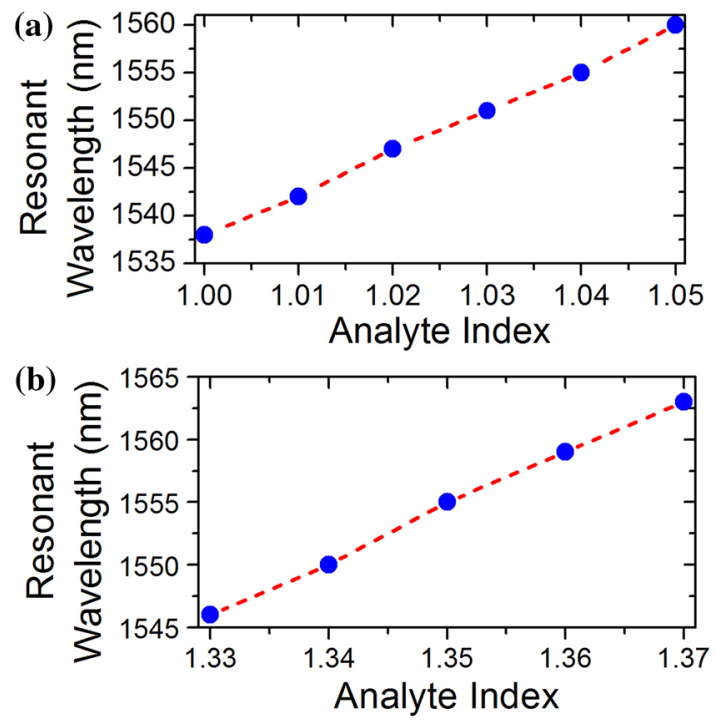

Fig. 10 Wavelength position shift with the analyte index, the slope of the curve denotes the sensitivity in nm/RIU. a Gas sensing. b Water sensing

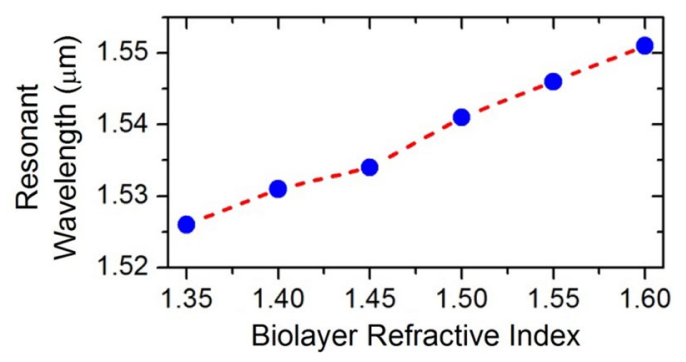

Fig. 11 Wavelength position shift with the biolayer refractive index, the slope of the curve denotes the sensitivity in nm/RIU. a Gas sensing. b Water sensing

biosensors [9], plasmonic biosensors [24], and photonic crystal biosensors [25].

In this section we investigate the capability of our silicon nanowire sensor to function as a biosensor with thin biolayers attached on the surface of the SiNWs. We simulate the biosensing situation where a thin biomaterial layer of only $10 \mathrm{~nm}$ thickness [26] gets attached to the surface of the SiNWs of our proposed sensor. The refractive index of such biolayer was varied from 1.35 to 1.60 to account for a number of biomaterials in that index range such as the $n=1.47$ bovine serum albumin [27] and the $n=1.5$ biotin-streptavidin [28]. Figure 11 shows the variation of the resonant wavelength of the ring resonator SiNW sensor with the index of the $10 \mathrm{~nm}$ thick biolayer.

The slope of the curve of Fig. 11 reveals that our sensor has a sensitivity of $100 \mathrm{~nm} / \mathrm{RIU}$ for a $10 \mathrm{~nm}$ thick biolayer. 


\section{Conclusion}

Silicon nanowire-based optical sensor has been developed. The sensor utilizes the ring resonator configuration. Critical coupling to the resonator as well the Q-factor of the resonator were studied. The spectrum of the resonator showed red-shift with the increased analyte index. The sensor was characterized by its low insertion loss of $0.4 \mathrm{~dB}$, and high sensitivity of $430 \mathrm{~nm} / \mathrm{RIU}$. Furthermore, the functionality of the sensor as a biosensor was investigated by attaching a biolayer of a small thickness of $10 \mathrm{~nm}$ to the silicon nanowires, the sensitivity measured was $100 \mathrm{~nm} / \mathrm{RIU}$ for such a small thickness.

Acknowledgements Open Access funding provided by the Qatar National Library. This work was made possible by a NPRP award [NPRP7456-1-085] from the Qatar National Research Fund (member of the Qatar Foundation). The statements made herein are solely the responsibility of the authors.

Open Access This article is distributed under the terms of the Creative Commons Attribution 4.0 International License (http://creativeco mmons.org/licenses/by/4.0/), which permits unrestricted use, distribution, and reproduction in any medium, provided you give appropriate credit to the original author(s) and the source, provide a link to the Creative Commons license, and indicate if changes were made.

\section{References}

1. W. Bogaerts et al., J. Lightwave Technol. 23, 1 (2005)

2. W. Bogaerts et al., Silicon-on-insulator spectral filters fabricated with CMOS technology. IEEE J Sel Top Quantum Electron 16, 1 (2010)

3. G.T. Reed, A.P. Knights, Silicon photonics: an introduction (Wiley, Hoboken, NJ, USA, 2004)

4. S.A. Maier, Plasmonics: fundamentals and applications (Springer, Berlin, 2007)

5. S.A. Maier, H.A. Atwater, J. Appl. Phys. 98, 1 (2005)

6. M. Khorasaninejad, S.S. Saini, Opt. Express 18, 22 (2010)

7. M. Khorasaninejad, M.A. Swillam, K. Pillai, S.S. Saini, Opt Lett 37, 20 (2012)

8. J.A. Dionne, L.A. Sweatlock, H.A. Atwater, A. Polman, Phys Rev B 73, 3 (2006)
9. R. Gamal, Y. Ismail, M.A. Swillam, J. Opt. 17, 4 (2015)

10. S.M. Sherif, D.C. Zografopoulos, L.A. Shahada, R. Beccherelli, M. Swillam, J. Phys. D: Appl. Phys. 50, 5 (2017)

11. M. Fox, Optical properties of solids (Oxford University Press, Oxford, 2011)

12. L.C. Kimerling, D. Ahn, A. B. Apsel, M. Beals, D. Carothers, Y. K. Chen, T. Conway, D. M. Gill, M. Grove, C. Y. Hong, M. Lipson, Electronic-photonic integrated circuits on the CMOS platform. in Proc. SPIE 6125, Silicon Photonics, 612502 (2006)

13. S. Mohamed, L. Shahada, M. Swillam, I.E.E.E. Photon, Technol. Lett. 30, 9 (2018)

14. M. Khorasaninejad, N. Clarke, M.P. Anantram, S.S. Saini, Opt. Express 19, 18 (2011)

15. M. Khorasaninejad, S.S. Saini, IEEE Photon J 3, 4 (2011)

16. Lumerical MODE Solutions, version 7.7.736, Lumerical Solutions Inc., https://www.lumerical.com/tcad-products/mode/ (2018)

17. Lumerical FDTD Solutions, version 8.16.982, Lumerical Solutions Inc., https://www.lumerical.com/tcad-products/fdtd/ (2018)

18. B. Fuhrmann, H.S. Leipner, H.R. Höche, L. Schubert, P. Werner, U. Gösele, Nano Lett. 5, 12 (2005)

19. Y.Q. Fu, A. Colli, A. Fasoli, J.K. Luo, A.J. Flewitt, A.C. Ferrari, W.I. Milne, J Vac Sci Technol B Microelectron Nanometer Struct. Process Meas Phenom 27, 3 (2009)

20. M.Y. Elsayed, A.M. Gouda, Y. Ismail, M.A. Swillam, J. Lightwave Technol. 35, 14 (2017)

21. A.M. Gouda, N.K. Allam, M.A. Swillam, RSC Adv. 7, 43 (2017)

22. S.A. Razek, M.A. Swillam, N.K. Allam, J. Appl. Phys. 115, 19 (2014)

23. S. Magdi, J. El-Rifai, M.A. Swillam, ACS Appl. Nano Mater. 1, $6(2018)$

24. M.Y. Azab, M.F.O. Hameed, A.M. Heikal, A.A. Swillam, S.S.A. Obayya, Design considerations of highly efficient D-shaped plasmonic biosensor. Opt. Quant. Electron. 51, 1 (2019)

25. S. Robinson, N. Dhanlaksmi, Photonic crystal based biosensor for the detection of glucose concentration in urine. Photon. Sens. 7, 3 (2017)

26. G.N. Tsigaridas, A study on refractive index sensors based on optical micro-ring resonators. Photon. Sens. 7, 3 (2017)

27. G.M. Hale, M.R. Querry, Optical constants of water in the 200-nm to $200-\mu \mathrm{m}$ wavelength region. Appl. Opt. 12, 3 (1973)

28. N. Skivesen, A. Têtu, M. Kristensen, J. Kjems, L.H. Frandsen, P.I. Borel, Photonic-crystal waveguide biosensor. Opt. Express. 15, 6 (2007)

Publisher's Note Springer Nature remains neutral with regard to jurisdictional claims in published maps and institutional affiliations. 